\title{
Complex magnetic fields: An improved Hardy-Laptev-Weidl inequality and quasi-self-adjointness
}

\author{
David Krejčiř́́k \\ Department of Mathematics, Faculty of Nuclear Sciences and Physical Engineering, Czech Technical \\ University in Prague, Trojanova 13, 12000 Prague 2, Czech Republic; david.krejcirik@fjfi.cvut.cz.
}

15 February 2018

\begin{abstract}
We show that allowing magnetic fields to be complex-valued leads to an improvement in the magnetic Hardy-type inequality due to Laptev and Weidl. The proof is based on the study of momenta on the circle with complex magnetic fields, which is of independent interest in the context of $\mathcal{P} \mathcal{T}_{\text {-symmetric }}$ and quasi-Hermitian quantum mechanics. We study basis properties of the non-self-adjoint momenta and derive closed formulae for the similarity transforms relating them to self-adjoint operators.
\end{abstract}

\section{Introduction}

The magnetic field in quantum mechanics is an appealing concept for several reasons. First, it enters the theory indirectly, through its vector potential in the Schrödinger equation, leading thus to purely quantum phenomena such as the Aharonov-Bohm effect. Second, the magnetic Hamiltonian is not invariant under complex conjugation, which results in the possibility of degeneracy of the ground state and other abnormal properties with respect to the theory of elliptic partial differential equations with real coefficients. Finally, the aesthetic dimension of the theory is also to be emphasised, for it can be described by a mathematically elegant, geometric framework of differential forms. Instead of choosing a few works from the huge bibliography devoted to the magnetic field in mathematical physics over the last hundred years, we refer to the recent Raymond's monograph [25], initially entitled "Little magnetic book", with many references.

In this paper we point out some new phenomena related to magnetic fields which are allowed to be complex-valued. It is remarkable that there is an experimental evidence for imaginary magnetic fields in quantum statistical physics [24, 1]. However, our primary motivations come from more theoretical aspects in quantum mechanics that we explain now.

\subsection{Improved magnetic Hardy inequalities}

Hardy inequalities are sometimes interpreted as a sort of uncertainty principle in quantum mechanics. More specifically, they are functional inequalities quantifying positivity of elliptic operators. For instance, the spectrum of the free Hamiltonian in $\mathbb{R}^{d}$, i.e. the self-adjoint realisation of the Laplacian in $L^{2}\left(\mathbb{R}^{d}\right)$, is stable against small short-range perturbations if, and only if, a Hardy inequality holds, that is, $d \geq 3$. It is a noteworthy observation of Laptev and Weidl [22] that Hardy inequalities hold even in the twodimensional situation provided that magnetic fields are added.

Our first observation is that complex "magnetic fields" lead to improved variants of the Hardy-type inequality due to Laptev and Weidl 22 in the real case.

Theorem 1. Let a smooth complex field $B: \mathbb{R}^{2} \rightarrow \mathbb{C}$ be compactly supported and satisfy the condition

$$
\frac{1}{2 \pi} \int_{\mathbb{R}^{2}} \Re B(x) \mathrm{d} x \notin \mathbb{Z} \quad \text { or } \quad \frac{1}{2 \pi} \int_{\mathbb{R}^{2}} \Im B(x) \mathrm{d} x \neq 0 .
$$


Then there exists a positive constant $c$ depending on $B$ such that the following inequality holds

$$
\forall \psi \in C_{0}^{\infty}\left(\mathbb{R}^{2}\right), \quad \int_{\mathbb{R}^{2}}\left|\nabla_{A} \psi(x)\right|^{2} \mathrm{~d} x \geq c \int_{\mathbb{R}^{2}} \frac{|\psi(x)|^{2}}{1+|x|^{2}} \mathrm{~d} x,
$$

where $\nabla_{A}:=\nabla-i A$ with

$$
A(x):=\left(-x_{2}, x_{1}\right) \int_{0}^{1} B(t x) t \mathrm{~d} t .
$$

In the sentence preceding the theorem, we have used quotation marks because the left-hand side of (2) is a symmetric quadratic form, associated with a self-adjoint operator in $L^{2}\left(\mathbb{R}^{2}\right)$, even if the vector potential $A$ is complex-valued. In other words, the differential expression $\left(\nabla_{A}\right)^{*} \nabla_{A}$ is of course formally self-adjoint, while the magnetic Hamiltonian would rather correspond to $-\nabla_{A} \nabla_{A}$, which is nonself-adjoint whenever $A$ is not real-valued. Hence, Theorem 1 is potentially relevant in the context of conventional quantum mechanics, where the "imaginary part of the magnetic field" typically relates the problem to a self-adjoint Schrödinger operator in a weighted $L^{2}$-space ( $c f$. Remark 1 below).

For $\Im B=0$, inequality (2) is the celebrated result of Laptev and Weidl [22, Thm. 1] and (11) reduces to the standard flux condition. The novelty here is that, for complex magnetic fields, one can get a nontrivial Hardy inequality even if the first condition of (11) is not satisfied. Indeed, it is enough that the magnetic field satisfies the second condition of (1), which is a kind of complex extension of the standard flux condition.

The relationship (3) ensures that $B=\operatorname{rot} A:=\partial_{1} A_{2}-\partial_{2} A_{1}$ and that the transverse condition

$$
x \cdot A(x)=0 \text {. }
$$

holds for all $x \in \mathbb{R}^{2}$. In view of the gauge invariance for real-valued $B$, the requirement (3) is superfluous and (2) holds for any smooth vector potential $A$ satisfying $B=\operatorname{rot} A$. In the complex case, however, the choice (3) is an inevitable part of the theorem.

The restriction to two-dimensional magnetic fields in Theorem 1 is just for simplicity of the presentation. Higher-dimensional analogues of Theorem 1 can be derived quite straightforwardly by combining the ideas of the present paper with the methods of [4]. Notice also that for non-trivial real-valued $B$ the condition (11) is not needed provided that the weight $\left(1+|x|^{2}\right)^{-1}$ on the right-hand side of (2) is replaced by $\left(1+|x|^{2} \log ^{2}|x|\right)^{-1}$, see 4]. In this paper complex extensions of this robust result are also provided (see Theorems 4 and 5 below). On the other hand, Theorem 1 is sharp in the sense that (2) cannot hold with a positive constant $c$ provided that the condition (1) is violated (see Remark 5 below).

Finally, let us notice that a singular Hardy weight on the right-hand side of (2) is admissible provided that the vector potential is singular, too. As an illustration, we consider the complex Aharonov-Bohm potential

$$
A_{\infty}(x):=\left(-x_{2}, x_{1}\right) \frac{\alpha}{|x|^{2}}, \quad \text { where } \quad \alpha \in \mathbb{C} .
$$

It can be obtained from (3) by formally putting $B=2 \pi \alpha \delta$, where $\delta$ is the Dirac delta function.

Theorem 2. Let the complex vector potential $A_{\infty}: \mathbb{R}^{2} \rightarrow \mathbb{C}^{2}$ given by (5) satisfy the condition

$$
\Re \alpha \notin \mathbb{Z} \quad \text { or } \quad \Im \alpha \neq 0 .
$$

Then there exists a positive constant $c_{\infty}$ depending on $\alpha$ such that the following inequality holds

$$
\forall \psi \in C_{0}^{\infty}\left(\mathbb{R}^{2} \backslash\{0\}\right), \quad \int_{\mathbb{R}^{2}}\left|\nabla_{A_{\infty}} \psi(x)\right|^{2} \mathrm{~d} x \geq c_{\infty} \int_{\mathbb{R}^{2}} \frac{|\psi(x)|^{2}}{|x|^{2}} \mathrm{~d} x .
$$

This theorem is a complex extension of another result of Laptev and Weidl [22, Thm. 3] in the real case. The new observation here is that the imaginary part of $\alpha$ leads to positivity even if its real part is an integer.

The proofs of Theorems 1 and 2 follow by extending the original ideas of 22 to the complex case. In some aspects we rather follow the more recent approach of [16] and 4]. The main idea is to use the transverse condition (4) and, by employing polar coordinates, reduce the problem to considering a magnetic Schrödinger operator on the circle. This leads us to another interesting aspect of complexvalued magnetic fields in quantum mechanics. 


\subsection{Quasi-self-adjointness of momenta with complex magnetic fields}

Many sustained attempts have been made by physicists in recent years to represent observables in quantum mechanics by operators which satisfy certain physical-like symmetries instead of the self-adjointness. This "extension" of quantum mechanics is often referred to as PT-symmetric quantum mechanics and its advent is usually associated with the pioneering work of Bender and Boettcher from 1998 [3]. The quotation marks are used here because nowadays it is commonly accepted that this unconventional representation of observables is consistent with fundamental axioms of quantum mechanics if, and only if, the non-self-adjoint representative $P$ is quasi-self-adjoint, i.e.

$$
P^{*}=\Theta P \Theta^{-1}
$$

with some positive, bounded and boundedly invertible operator $\Theta$ called metric (the special choice $\Theta=I$ corresponds to self-adjointness). But then one is back in the conventional quantum mechanics just by modifying the inner product $(\cdot, \cdot)$ in the underlying Hilbert space to $(\cdot, \Theta \cdot)$. The notion of quasi-selfadjoint (then called quasi-Hermitian) operators in quantum mechanics was first used by nuclear physicists Scholtz, Geyer and Hahne in 1992 [26], but it was actually considered previously by the mathematician Dieudonné as early as in 1961 [6]. We refer to the review article [19] and the book chapter [18] for mathematical aspects of quasi-self-adjoint quantum mechanics.

Given a non-self-adjoint operator $P$ with real spectrum, it is usually not easy to decide whether it is quasi-self-adjoint. Even if the latter is known to hold for an operator $P$ (like for instance for operators whose eigenfunctions form a Riesz basis), one cannot expect to be able to solve the operator equation (8) and find the metric operator $\Theta$ in a closed form. Distinguished exceptions are represented by onedimensional Schrödinger operators with non-self-adjoint point interactions on intervals [17, 15, 27, 17, 9, 8, 20] or graphs [13, 2, 21]. We particularly refer to [20] where the general study of Sturm-Liouville operators with complex Robin boundary conditions reveals that the metric can be expressed as the sum of the identity and a Hilbert-Schmidt operator, reflecting the Bari basis property of the eigenfunctions. Moreover, spectacularly simple formulae of the integral kernels are provided in specific $(\mathcal{P} \mathcal{T}$-symmetric) situations.

The second motivation of this paper comes from the relevance of complex magnetic fields in these nonself-adjoint representations in quantum mechanics. Papers on the imaginary magnetic field in quantum mechanics exist in the literature (see, e.g., [1, 12]), but the quasi-self-adjointness does not seem to have been considered. In this paper we introduce momenta with complex magnetic fields, as a new class of non-self-adjoint toy models, which are probably the simplest non-trivial examples of quasi-self-adjoint operators in infinite-dimensional Hilbert spaces whatsoever. It turns out that the basis properties of the eigenfunctions substantially differ from the aforementioned models with point interactions. Because of the technical relationship with the magnetic Hardy inequalities, we restrict to momenta on the unit circle $\mathbb{S} \cong(-\pi, \pi)$, but analogous models can be considered on intervals (even unbounded) and graphs.

Theorem 3. Given $a \in L^{2}(\mathbb{S} ; \mathbb{C})$, let $P_{a}$ be the maximal realisation of the operator

$$
-i \frac{\mathrm{d}}{\mathrm{d} x}-a(x) \quad \text { in } \quad L^{2}(\mathbb{S}) .
$$

The operator $P_{a}$ satisfies the following properties:

(i) The similarity relation

$$
\Omega_{a} P_{a} \Omega_{a}^{-1}=P_{\langle a\rangle}
$$

holds, where

$$
\left(\Omega_{a} \psi\right)(x):=\exp \left(i\langle a\rangle x-i \int_{-\pi}^{x} a(\xi) \mathrm{d} \xi\right) \psi(x) \quad \text { with } \quad\langle a\rangle:=\frac{1}{2 \pi} \int_{-\pi}^{\pi} a(x) \mathrm{d} x .
$$

(ii) The operator $P_{a}$ is quasi-self-adjoint if, and only if,

$$
\langle\Im a\rangle=0 \text {. }
$$

In this case, $P_{a}$ satisfies the relation (8) with the metric given by the multiplication operator

$$
\left(\Theta_{a} \psi\right)(x):=\exp \left(2 \int_{-\pi}^{x} \Im a(\xi) \mathrm{d} \xi\right) \psi(x) .
$$


(iii) The eigenfunctions of $P_{a}$ form a Riesz basis, but not a Bari basis unless $\Im a=0$.

We emphasise that the similarity relation (10) holds in general, in particular without assuming (12), but the transformed operator $P_{\langle a\rangle}$ is self-adjoint if, and only if, this condition holds. Condition (12) is a complex extension of the requirement that the magnetic field on the circle can be gauged out. Notice also ( $c f .(17)$ ) that $P_{a}$ is self-adjoint if, and only if, $\Im a=0$, while (12) can be of course satisfied in more general situations.

\section{Momenta with complex magnetic fields}

In this section we restrict to the one-dimensional model (9). We identify the unit circle $\mathbb{S}$ with the open interval $(-\pi, \pi)$ where the boundary points $\pm \pi$ are glued together.

\subsection{Definition}

We introduce $P_{a}$ as the operator in $L^{2}((-\pi, \pi))$ defined by

$$
\left(P_{a} \psi\right)(x):=-i \psi^{\prime}(x)-a(x) \psi(x), \quad \operatorname{dom} P_{a}:=\left\{\psi \in W^{1,2}((-\pi, \pi)): \psi(-\pi)=\psi(\pi)\right\} .
$$

Our standing assumption is that the function $a:(-\pi, \pi) \rightarrow \mathbb{C}$ representing a complex-valued magnetic potential is square-integrable, i.e.,

$$
a \in L^{2}((-\pi, \pi))
$$

In the magnetic-free case, $P_{0}$ is the usual momentum operator in quantum mechanics, which is well known to be self-adjoint.

Under the hypothesis (15), the magnetic part of $P_{a}$ is a small perturbation of $P_{0}$. Indeed, for every $\psi \in W^{1,2}((-\pi, \pi))$ and positive $\varepsilon$, we have the bound

$$
\|a \psi\|^{2} \leq\|a\|^{2}\|\psi\|_{\infty}^{2} \leq \varepsilon\|a\|^{2}\left\|\psi^{\prime}\right\|^{2}+\|a\|^{2}\left(\frac{1}{\varepsilon}+\frac{1}{2 \pi}\right)\|\psi\|^{2},
$$

where $\|\cdot\|$ is the norm of $L^{2}((-\pi, \pi))$ and $\|\cdot\|_{\infty}$ is the supremum norm. Here the second inequality is due to the Sobolev embedding $W^{1,2}((-\pi, \pi)) \hookrightarrow C^{0}([-\pi, \pi])$ quantified by

$$
\|\psi\|_{\infty}^{2} \leq 2\|\psi\|\left\|\psi^{\prime}\right\|+\frac{1}{2 \pi}\|\psi\|^{2}
$$

and an elementary Young-type inequality. It follows from (16) that $a$, considered as the multiplication operator in $L^{2}((-\pi, \pi))$, is relatively bounded with respect to $P_{0}$, with the relative bound equal to zero. By standard perturbation results [14, Sec. IV.1], $P_{a}$ is a well defined closed operator with compact resolvent.

Remark 1. Let $\psi \in W^{1,2}((-\pi, \pi))$ and consider the changed function

$$
\phi(x):=w(x) \psi(x) \quad \text { with } \quad w(x):=\exp \left(\int_{-\pi}^{x} \Im a(\xi) \mathrm{d} \xi\right) .
$$

In view of the obvious relationship between quadratic forms

$$
\int_{-\pi}^{\pi}\left|\left(P_{a} \psi\right)(x)\right|^{2} \mathrm{~d} x=\int_{-\pi}^{\pi}\left|\left(P_{\Re a} \psi\right)(x)\right|^{2} w^{-2}(x) \mathrm{d} x,
$$

one can interpret the imaginary part of the magnetic field as a way to handle the conventional (selfadjoint) magnetic Schrödinger operator in a weighted space. Notice also that $\phi \in \operatorname{dom} P_{a}$ provided that $\psi \in \operatorname{dom} P_{a}$ and the condition (12) holds. 


\subsection{Obvious symmetries}

The adjoint of $P_{a}$ satisfies

$$
P_{a}^{*}=P_{\bar{a}}
$$

where $\bar{a}$ denotes the complex conjugate of $a$. Consequently, $P_{a}$ is self-adjoint if, and only if, the imaginary part of $a$ identically equals zero, i.e. $\Im a=0$.

Let the time-reversal operator $\mathcal{T}$ and parity operator $\mathcal{P}$ be defined by the usual involutions

$$
(\mathcal{T} \psi)(x):=\overline{\psi(x)} \quad \text { and } \quad(\mathcal{P} \psi)(x):=\psi(-x) .
$$

Then we obviously have the relations

$$
\mathcal{T} P_{a} \mathcal{T}=-P_{-\mathcal{T} a}, \quad \mathcal{P} P_{a} \mathcal{P}=-P_{-\mathcal{P} a} \quad \text { and } \quad(\mathcal{P T}) P_{a}(\mathcal{P} \mathcal{T})=P_{\mathcal{P} \mathcal{T} a}
$$

Consequently, $P_{a}$ is anti- $\mathcal{P}$-self-adjoint $\left(i . e ., P_{a}^{*}=-\mathcal{P} P_{a} \mathcal{P}\right)$ if, and only if, $\Re a$ is odd and $\Im a$ is even; and $P_{a}$ is $\mathcal{P} \mathcal{T}$-symmetric (i.e., $\left[P_{a}, \mathcal{P T}\right]=0$ ) if, and only if, $\Re a$ is even and $\Im a$ is odd.

The $\mathcal{P} \mathcal{T}$-symmetry of $P_{a}$ is a sufficient (but not necessary) condition to guarantee the quasi-selfadjointness condition (12).

Remark 2. Instead of the momentum operator $P_{a}$, one can also consider the non-self-adjoint magnetic Hamiltonian $H_{a}:=P_{a}^{2}$. Then it follows that $H_{a}$ is $\mathcal{P}$-self-adjoint (i.e., $\left.H_{a}{ }^{*}=\mathcal{P} H_{a} \mathcal{P}\right)$ if, and only if, $\Re a$ is odd and $\Im a$ is even; and $H_{a}$ is $\mathcal{P} \mathcal{T}$-symmetric if, and only if, $\Re a$ is even and $\Im a$ is odd. This is an example of operator where these two notions are not compatible unless $a=0$. At the same time, $H_{a}$ is

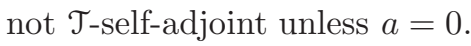

\subsection{Spectrum}

Since $P_{a}$ has a compact resolvent, its spectrum is purely discrete, i.e. composed of isolated eigenvalues with finite algebraic multiplicities. In this one-dimensional setting, finding the eigenvalues is a routine: solving the differential equation $-i \psi^{\prime}-a(x) \psi=\lambda \psi$ in terms of exponential functions and subjecting the solutions to the periodic boundary conditions of (14), one immediately arrives at

$$
\sigma\left(P_{a}\right)=\{m-\langle a\rangle\}_{m \in \mathbb{Z}}
$$

Notice that the spectrum is real if, and only if, the quasi-self-adjointness condition (12) holds. Again, the $\mathcal{P} \mathcal{T}$-symmetry of $P_{a}$ is a sufficient (but not necessary) condition to guarantee that the spectrum is real. Irrespectively of whether $P_{a}$ is $\mathcal{P} \mathcal{T}$-symmetric or not, the spectrum of the adjoint $P_{a}^{*}$ is obtained by mirroring the eigenvalues of $P_{a}$ with respect to the real axis.

By the procedure described above, one also finds the eigenfunctions of $P_{a}$ corresponding to the eigenvalues in (18),

$$
\psi_{m}(x):=\frac{1}{\sqrt{2 \pi}} \exp \left(i(m-\langle a\rangle) x+i \int_{-\pi}^{x} a(\xi) \mathrm{d} \xi\right) .
$$

The eigenfunctions of the adjoint $P_{a}^{*}$ are given by

$$
\phi_{m}(x):=\frac{1}{\sqrt{2 \pi}} \exp \left(i(m-\langle\bar{a}\rangle) x+i \int_{-\pi}^{x} \bar{a}(\xi) \mathrm{d} \xi\right)
$$

The normalisation factors are chosen in such a way that the standard biorthogonal condition

$$
\left(\phi_{n}, \psi_{m}\right)=\delta_{n m}
$$

holds for every $m, n \in \mathbb{Z}$, where $(\cdot, \cdot)$ denotes the inner product of $L^{2}((-\pi, \pi))$. This condition reduces to $\left(\psi_{n}, \psi_{m}\right)=\delta_{n m}$ if $\Im a=0$ (so that $P_{a}$ is self-adjoint).

The availability of the condition (19) ensures that the spectrum of $P_{a}$ is semisimple (i.e. the algebraic and geometric multiplicities of the eigenvalues are equal). From (18) we then immediately conclude that the spectrum is actually simple (i.e. all the eigenvalues have multiplicity one). 


\subsection{Basis properties}

For various basis properties of a sequence in a Hilbert space, we refer to [10] and [5. Sec. 3].

The principal observation in our case is that, for every $m \in \mathbb{Z}$,

$$
\psi_{m}(x)=\xi(x) e_{m}(x) \quad \text { and } \quad \phi_{m}(x)=\xi^{-1}(x) e_{m}(x)
$$

where

$$
\xi(x):=\exp \left(\langle\Im a\rangle x-\int_{-\pi}^{x} \Im a(\xi) \mathrm{d} \xi\right)
$$

is a bounded and positive function on $[-\pi, \pi]$, while $\left\{e_{m}\right\}_{m \in \mathbb{Z}}$ is an orthonormal basis in $L^{2}((-\pi, \pi))$ (for it corresponds to the choice $\Im a=0$ when $P_{a}$ is self-adjoint). Consequently, it immediately follows that the sequence $\left\{\psi_{m}\right\}_{m \in \mathbb{Z}}$ is almost normalised in $L^{2}((-\pi, \pi))$ in the sense that

$$
\inf _{m \in \mathbb{Z}}\left\|\psi_{m}\right\|>0 \quad \text { and } \quad \sup _{m \in \mathbb{Z}}\left\|\psi_{m}\right\|<\infty .
$$

Next, assuming $\left(\psi_{m}, \psi\right)=0$ for every $m \in \mathbb{Z}$ and using the completeness of $\left\{e_{m}\right\}_{m \in \mathbb{Z}}$, we conclude that $\xi \psi=0$, and therefore $\psi=0$. This argument shows that $\left\{\psi_{m}\right\}_{m \in \mathbb{Z}}$ is a complete set in $L^{2}((-\pi, \pi))$. In view of (19), we also know that $\left\{\psi_{m}\right\}_{m \in \mathbb{Z}}$ is minimal complete (i.e. removal of any element makes it incomplete).

Similarly, since $\left\{e_{m}\right\}_{m \in \mathbb{Z}}$ is in particular a (Schauder or conditional) basis, given any function $\psi \in$ $L^{2}((-\pi, \pi))$, we have the unique decomposition

$$
\psi=\sum_{m \in \mathbb{Z}}\left(e_{m}, \psi\right) e_{m}=\sum_{m \in \mathbb{Z}}\left(\xi^{-1} e_{m}, \psi\right) \xi e_{m} .
$$

Using (20), we conclude that $\left\{\psi_{m}\right\}_{m \in \mathbb{Z}}$ is a basis in $L^{2}((-\pi, \pi))$.

In fact, having the completeness of $\left\{\psi_{m}\right\}_{m \in \mathbb{Z}}$, we immediately conclude from (20) that $\left\{\psi_{m}\right\}_{m \in \mathbb{Z}}$ is a Riesz (or unconditional) basis in $L^{2}((-\pi, \pi)$ ), for it is obviously equivalent to an orthonormal basis (cf. [5, Thm. 3.4.5]). This shows one part of the claim (iii) of Theorem 3. Because of the biorthogonality relation (19), we also know that $\left\{\phi_{m}\right\}_{m \in \mathbb{Z}}$ is a Riesz basis in $L^{2}((-\pi, \pi))$.

It remains to prove that $\left\{\psi_{m}\right\}_{m \in \mathbb{Z}}$ is not a Bari basis if $\Im a \neq 0$, meaning that it is not quadratically close to any orthonormal basis. By [10, Sec. VI.3], it is enough to show that the biorthogonal bases $\left\{\psi_{m}\right\}_{m \in \mathbb{Z}}$ and $\left\{\phi_{m}\right\}_{m \in \mathbb{Z}}$ are not quadratically close to each other. Using that $\left|e_{m}\right|=1 / \sqrt{2 \pi}$ for every $m \in \mathbb{Z}$ and (20), we arrive at $\left|\psi_{m}-\phi_{m}\right|=\left|\xi-\xi^{-1}\right| / \sqrt{2 \pi}$ which is independent of $m$. Consequently,

$$
\sum_{m \in \mathbb{Z}}\left\|\psi_{m}-\phi_{m}\right\|^{2}=\infty
$$

unless $\xi=\xi^{-1}$, which is possible only if $\Im a=0$. This concludes the proof of the claim (iii) of Theorem 3

\subsection{Hidden symmetries}

Now we turn to the proof of the remaining claims of Theorem 3 .

First of all, notice that $\Omega_{a}$ from the claim (i) of Theorem 3 is a multiplication operator generated by a bounded and non-zero differentiable function on $[-\pi, \pi]$. A formal verification of the similarity relation (10) is a routine. To make it rigorous, it remains to show that $\Omega_{a}$ leaves the domain of $P_{a}$ (which is independent of $a$, see (14) ) invariant. The principal step is to verify the invariance of the periodic boundary conditions. Given any $\psi \in W^{1,2}((-\pi, \pi))$, it is straightforward to check (recall also (16) ) that $\Omega_{a} \psi \in W^{1,2}((-\pi, \pi))$ and

$$
\left(\Omega_{a} \psi\right)(-\pi)=e^{-i\langle a\rangle \pi} \psi(-\pi), \quad\left(\Omega_{a} \psi\right)(\pi)=e^{i\langle a\rangle \pi} e^{-i 2 \pi\langle a\rangle} \psi(-\pi) .
$$

Hence, if $\psi$ additionally satisfies $\psi(\pi)=\psi(-\pi)$, then it is also the case for $\Omega_{a} \psi$. Summing up, we have verified the claim (i) of Theorem 3 .

Now we turn to the claim (ii) of Theorem 3. The operator $P_{\langle a\rangle}$ is self-adjoint if, and only if, the condition (12) holds. In this case, $P_{a}$ is similar to a self-adjoint operator in view of (10) and it clearly satisfies the relation (8) with the metric operator $\Theta_{a}=\Omega_{a}^{*} \Omega_{a}$, which coincides with formula (13). Conversely, 
if $P_{a}$ satisfies the relation (8) with some positive, bounded and boundedly invertible operator $\Theta$, then it is clearly similar to the self-adjoint operator $\Theta^{1 / 2} P_{a} \Theta^{-1 / 2}$. Hence, the spectrum of $P_{a}$ is necessarily real, which is equivalent to the condition (12) due to the explicit formula (18). So we are back in the situation where $P_{\langle a\rangle}$ is self-adjoint. This concludes the proof of Theorem 3 .

Remark 3. Irrespectively of whether the condition (12) holds or not, $P_{a}$ is always similar to the normal operator $P_{\langle a\rangle}$.

\section{$3 \quad$ Hardy inequalities with complex magnetic fields}

This section is devoted to proofs of Theorems 1 and 2 from the introduction and other related results. The standing assumption for regular fields is that the function $B: \mathbb{R}^{2} \rightarrow \mathbb{C}$ representing a complex magnetic field is (infinitely) smooth, i.e. $B \in C^{\infty}\left(\mathbb{R}^{2}\right)$. For the proof of Theorem 1 we shall additionally assume that $B$ has a compact support, i.e. $B \in C_{0}^{\infty}\left(\mathbb{R}^{2}\right)$. It will be clear from the proof below that much less is actually needed to establish Theorem 1, Since $B$ is complex-valued, the freedom of the gauge invariance is lost and we stick (except for Section 3.5) to the choice of the corresponding vector potential $A: \mathbb{R}^{2} \rightarrow \mathbb{C}^{2}$ given by (3). Because of the smoothness assumption about the field $B$, we then also have $A \in C^{\infty}\left(\mathbb{R}^{2} ; \mathbb{C}^{2}\right)$.

\subsection{Passing to polar coordinates}

The main idea coming back to 22] (see also [16] and 44) is to employ polar coordinates $x=\left(x_{1}, x_{2}\right)=$ $(r \cos \theta, r \sin \theta)$ with $x \in \mathbb{R}^{2}$ and $r \in(0, \infty), \theta \in(-\pi, \pi)$. Then (3) acquires a more transparent form

$$
A(x)=(-\sin \theta, \cos \theta) \frac{a(r, \theta)}{r}, \quad \text { where } \quad a(r, \theta):=\int_{0}^{r} B(t \cos \theta, t \sin \theta) t \mathrm{~d} t .
$$

In particular, we have $a \in C^{\infty}((0, \infty) \times[-\pi, \pi])$.

Recall the mean-value notation $\langle\cdot\rangle$ introduced in (11). If $B$ has a compact support, then there clearly exists a positive number $R$ such that $\langle a(r, \cdot)\rangle=\langle a(R, \cdot)\rangle$ for all $r \geq R$. The flux condition (1) is then equivalent to

$$
\lim _{r \rightarrow \infty}\langle\Re a(r, \cdot)\rangle \notin \mathbb{Z} \quad \text { or } \quad \lim _{r \rightarrow \infty}\langle\Im a(r, \cdot)\rangle \neq 0 .
$$

Occasionally, we shall also consider the weaker condition

$$
\exists r \in(0, \infty), \quad\langle\Re a(r, \cdot)\rangle \notin \mathbb{Z} \quad \text { or } \quad\langle\varsigma a(r, \cdot)\rangle \neq 0,
$$

for which we do not need to assume that $B$ is compactly supported.

Let $\psi \in C_{0}^{\infty}\left(\mathbb{R}^{2}\right)$. Writing $\phi(r, \theta):=\psi(r \cos \theta, r \sin \theta)$ and using the transverse property (4) of $A$, we have

$$
\int_{\mathbb{R}^{2}}\left|\nabla_{A} \psi(x)\right|^{2} \mathrm{~d} x=\int_{0}^{\infty} \int_{-\pi}^{\pi}\left(\left|\partial_{r} \phi(r, \theta)\right|^{2}+\frac{\left|-i \partial_{\theta} \phi(r, \theta)-a(r, \theta) \phi(r, \theta)\right|^{2}}{r^{2}}\right) \mathrm{d} \theta r \mathrm{~d} r .
$$

Recalling (14), it is clear that the concept of momenta on the circle with complex magnetic fields developed in the precedent section will play an important role in the sequel.

\subsection{Local Hardy inequalities}

For every positive $r$, let us define a non-negative number

$$
\lambda_{a}(r):=\inf _{\varphi \in \operatorname{dom}_{\varphi \neq 0} P_{a}} \frac{\int_{-\pi}^{\pi}\left|\left(P_{a(r, \cdot)} \varphi\right)(\theta)\right|^{2} \mathrm{~d} \theta}{\int_{-\pi}^{\pi}|\varphi(\theta)|^{2} \mathrm{~d} \theta},
$$

where dom $P_{a}$ is introduced in (14). Clearly, $\lambda_{a}(r)$ is the lowest point in the spectrum of the self-adjoint operator $P_{a(r, \cdot)}{ }^{*} P_{a(r, \cdot)}$ in $L^{2}((-\pi, \pi))$, where $r$ is regarded as a parameter. If $B$ has a compact support, then there clearly exists a positive number $R$ such that $\lambda_{a}(r)=\lambda_{a}(R)$ for all $r \geq R$. 
Using the definition (25) in (24) together with Fubini's theorem, we arrive at the following type of Hardy inequality

$$
\begin{aligned}
\int_{\mathbb{R}^{2}}\left|\nabla_{A} \psi(x)\right|^{2} \mathrm{~d} x & \geq \int_{0}^{\infty} \int_{-\pi}^{\pi}\left(\left|\partial_{r} \phi(r, \theta)\right|^{2}+\frac{\lambda_{a}(r)}{r^{2}}|\phi(r, \theta)|^{2}\right) \mathrm{d} \theta r \mathrm{~d} r \\
& \geq \int_{0}^{\infty} \int_{-\pi}^{\pi} \frac{\lambda_{a}(r)}{r^{2}}|\phi(r, \theta)|^{2} \mathrm{~d} \theta r \mathrm{~d} r \\
& =\int_{\mathbb{R}^{2}} \frac{\lambda_{a}(|x|)}{|x|^{2}}|\psi(x)|^{2} \mathrm{~d} x .
\end{aligned}
$$

We remark ( $c f$. (21) ) that $\lambda_{a}(r)=\mathcal{O}\left(r^{2}\right)$ as $r \rightarrow 0$, so that $\lambda_{a}(r) / r^{2}$ has actually no singularity at $r=0$.

We call the result (26) a local Hardy inequality because $\lambda_{a}$ may not be everywhere positive. However, the following lemma ensures that $\lambda_{a}$ is a non-trivial non-zero function on $(0, \infty)$ whenever the condition (23) holds.

Lemma 1. The function $r \mapsto \lambda_{a}(r)$ is continuous. Given any $r \in(0, \infty), \lambda_{a}(r)=0$ if, and only if,

$$
\langle\Re a(r, \cdot)\rangle \in \mathbb{Z} \quad \text { and } \quad\langle\Im a(r, \cdot)\rangle=0 .
$$

Proof. Since dom $P_{a} \subset W^{1,2}((-\pi, \pi))$ is compactly embedded in $L^{2}((-\pi, \pi))$, the spectrum of the operator $P_{a(r, \cdot)}{ }^{*} P_{a(r, \cdot)}$ is purely discrete. Consequently, the infimum in 25] is achieved and $\lambda_{a}(r)$ is the lowest eigenvalue of this operator. Let us denote by $\varphi_{r}$ a corresponding eigenfunction.

First of all, we notice that, using a constant as a test function in (25), we have the upper bound

$$
\lambda_{a}(r) \leq\left\langle|a(r, \cdot)|^{2}\right\rangle .
$$

Using $\varphi_{r_{2}}$ as a test function for $\lambda_{a}\left(r_{1}\right)$, it follows from (25) that

$$
\lambda_{a}\left(r_{1}\right) \leq \frac{\int_{-\pi}^{\pi}\left|\left(P_{a\left(r_{2}, \cdot\right)} \varphi_{r_{2}}\right)(\theta)+\left[a\left(r_{2}, \theta\right)-a\left(r_{1}, \theta\right)\right] \varphi_{r_{2}}(\theta)\right|^{2} \mathrm{~d} \theta}{\int_{-\pi}^{\pi}\left|\varphi_{r_{2}}(\theta)\right|^{2} \mathrm{~d} \theta} \leq\left(\sqrt{\lambda_{a}\left(r_{2}\right)}+\sigma\left(r_{1}, r_{2}\right)\right)^{2}
$$

with

$$
\sigma\left(r_{1}, r_{2}\right):=\sup _{\theta \in(-\pi, \pi)}\left|a\left(r_{2}, \theta\right)-a\left(r_{1}, \theta\right)\right| .
$$

Using additionally the analogous estimate obtained by reversing the role of $r_{1}$ and $r_{2}$ together with (28), one eventually gets

$$
\left|\lambda_{a}\left(r_{2}\right)-\lambda_{a}\left(r_{1}\right)\right| \leq \sigma\left(r_{1}, r_{2}\right)^{2}+2 \sigma\left(r_{1}, r_{2}\right) \max \left\{\sqrt{\left\langle\left|a\left(r_{1}, \cdot\right)\right|^{2}\right.}, \sqrt{\left\langle\left|a\left(r_{2}, \cdot\right)\right|^{2}\right.}\right\} .
$$

By virtue of the smoothness of $a$, this inequality ensures that $\lambda_{a}$ is continuous (even Lipschitz).

Let us now fix any positive $r$ and show that (27) implies that $\lambda_{a}(r)=0$. Using the similarity relation (10), one has the estimate

$$
\lambda_{a}(r) \leq \kappa_{a}(r)^{2} \inf _{\substack{\varphi \operatorname{dom}_{\varphi \neq 0} \\ P_{a}}} \frac{\int_{-\pi}^{\pi}\left|\left(P_{\langle a(r, \cdot)\rangle} \Omega_{a(r, \cdot)} \varphi\right)(\theta)\right|^{2} \mathrm{~d} \theta}{\int_{-\pi}^{\pi}\left|\left(\Omega_{a(r, \cdot)} \varphi\right)(\theta)\right|^{2} \mathrm{~d} \theta}
$$

where the condition number $\kappa_{a}(r):=\left\|\Omega_{a(r, \cdot)}\right\|\left\|\Omega_{a(r, \cdot)}{ }^{-1}\right\|$ satisfies $1 \leq \kappa_{a}(r)<\infty$. Here $\|\cdot\|$ stands for the operator norm in $L^{2}((-\pi, \pi))$, which can be expressed through a supremum norm in our case for the operator in question is a multiplication operator. In view of the second condition of (27), the operator $P_{\langle a(r, \cdot)\rangle}$ is self-adjoint, and therefore the Rayleigh quotient in (29) equals the square of its smallest eigenvalue in absolute value. Recalling (18), we thus obtain

$$
\lambda_{a}(r) \leq \kappa_{a}(r)^{2} \operatorname{dist}(\langle\Re a(r, \cdot)\rangle, \mathbb{Z})^{2} .
$$


Using now in addition the first condition of (27), we conclude that $\lambda_{a}(r)=0$.

Conversely, assuming that $\lambda_{a}(r)=0$ for a given positive $r$, let us show that necessarily (27) holds. Indeed, if $\lambda_{a}(r)=0$, it follows from (25) that $P_{a(r, \cdot)} \varphi_{r}=0$, whence $0 \in \sigma\left(P_{a(r, \cdot)}\right)$. Recalling (18), the latter is possible only if (27) holds.

Remark 4. Proceeding as in (29) and (30), and assuming the second condition of (27), one also has the lower bound

$$
\lambda_{a}(r) \geq \kappa_{a}(r)^{-2} \operatorname{dist}(\langle\Re a(r, \cdot)\rangle, \mathbb{Z})^{2} .
$$

\subsection{From local to global Hardy inequalities}

Now we explain how to pass from the local Hardy inequality (26) to a global one (i.e. with a Hardy weight positive everywhere in $\mathbb{R}^{2}$ ). Notice that the following theorem does not require compactly supported magnetic fields and the weaker hypothesis (23) is assumed.

Theorem 4. Let a smooth complex field $B: \mathbb{R}^{2} \rightarrow \mathbb{C}$ satisfy the condition

$$
\exists r \in(0, \infty), \quad \frac{1}{2 \pi} \int_{D_{r}} \Re B(x) \mathrm{d} x \notin \mathbb{Z} \quad \text { or } \quad \frac{1}{2 \pi} \int_{D_{r}} \Im B(x) \mathrm{d} x \neq 0,
$$

where $D_{r}:=\left\{x \in \mathbb{R}^{2}:|x|<r\right\}$ is the disk of radius $r$. Then there exists a positive constant $\tilde{c}$ depending on $B$ such that the following inequality holds

$$
\forall \psi \in C_{0}^{\infty}\left(\mathbb{R}^{2}\right), \quad \int_{\mathbb{R}^{2}}\left|\nabla_{A} \psi(x)\right|^{2} \mathrm{~d} x \geq \tilde{c} \int_{\mathbb{R}^{2}} \frac{|\psi(x)|^{2}}{1+|x|^{2} \log ^{2}(|x|)} \mathrm{d} x,
$$

where the complex vector potential $A$ is given by (3).

Proof. The claim follows by mimicking the proof of 4 , Thm. 3.1]. Let $\psi \in C_{0}^{\infty}\left(\mathbb{R}^{2}\right)$ and recall that we denote by $\phi$ its counterpart in polar coordinates.

From Lemma 1 it follows that there exists a positive constant $\nu$ (depending on the behaviour of the function $\lambda_{a}$, which is in turn determined by the behaviour of $B$ ) and a bounded open interval $I \subset(0, \infty)$ such that $\lambda_{a}(r) / r^{2} \geq \nu>0$ for all $r \in I$. From (26) we thus conclude

$$
\int_{\mathbb{R}^{2}}\left|\nabla_{A} \psi(x)\right|^{2} \mathrm{~d} x \geq \nu \int_{\mathbb{R}^{2}} \chi_{I}(x)|\psi(x)|^{2} \mathrm{~d} x,
$$

where $\chi_{I}$ denotes the characteristic function of the annulus $\left\{x \in \mathbb{R}^{2}:|x| \in I\right\}$.

To extend this local Hardy inequality to $\mathbb{R}^{2}$, we employ the presence of the kinetic term that we neglected in (26), namely

$$
\int_{\mathbb{R}^{2}}\left|\nabla_{A} \psi(x)\right|^{2} \mathrm{~d} x \geq \int_{0}^{\infty} \int_{-\pi}^{\pi}\left|\partial_{r} \phi(r, \theta)\right|^{2} \mathrm{~d} \theta r \mathrm{~d} r
$$

together with the one-dimensional Hardy-type inequalities ( $c f$. [4, Lem. 3.1])

$$
\begin{aligned}
\forall f \in C_{0}^{\infty}\left(\mathbb{R} \backslash\left\{r_{0}\right\}\right), \quad & \int_{0}^{r_{0}}\left|f^{\prime}(r)\right|^{2} r \mathrm{~d} r \geq \gamma \int_{0}^{r_{0}}|f(r)|^{2} r \mathrm{~d} r, \\
& \int_{r_{0}}^{\infty}\left|f^{\prime}(r)\right|^{2} r \mathrm{~d} r \geq \gamma \int_{r_{0}}^{\infty} \frac{|f(r)|^{2}}{r^{2} \log ^{2}\left(r / r_{0}\right)} r \mathrm{~d} r,
\end{aligned}
$$

valid for any positive $r_{0}$ with some positive constant $\gamma$ depending only on $r_{0}$. Choosing $r_{0}$ to be the middle point of the interval $I$, we introduce a cut-off function $\xi \in C^{\infty}((0, \infty))$ such that $0 \leq \xi \leq 1, \xi$ vanishes in a neighbourhood of $r_{0}$ and $\xi=1$ outside the interval $I$. Writing $\phi(r, \theta)=\xi(r) \phi(r, \theta)+(1-\xi(r)) \phi(r, \theta)$ 
and using (35) with help of Fubini's theorem, we get

$$
\begin{aligned}
\int_{-\pi}^{\pi} & \int_{0}^{\infty} \frac{|\phi(r, \theta)|^{2}}{1+r^{2} \log ^{2}\left(r / r_{0}\right)} r \mathrm{~d} r \mathrm{~d} \theta \\
& \leq 2 \int_{-\pi}^{\pi} \int_{0}^{\infty} \frac{|\xi(r) \phi(r, \theta)|^{2}}{1+r^{2} \log ^{2}\left(r / r_{0}\right)} r \mathrm{~d} r \mathrm{~d} \theta+2 \int_{-\pi}^{\pi} \int_{0}^{\infty}|(1-\xi(r)) \phi(r, \theta)|^{2} r \mathrm{~d} r \mathrm{~d} \theta \\
& \leq \frac{2}{\gamma} \int_{-\pi}^{\pi} \int_{0}^{\infty}\left|\partial_{r}[\xi(r) \phi(r, \theta)]\right|^{2} r \mathrm{~d} r \mathrm{~d} \theta+2 \int_{-\pi}^{\pi} \int_{I}|\phi(r, \theta)|^{2} r \mathrm{~d} r \mathrm{~d} \theta \\
& \leq \frac{4}{\gamma} \int_{-\pi}^{\pi} \int_{0}^{\infty}\left|\partial_{r} \phi(r, \theta)\right|^{2} r \mathrm{~d} r \mathrm{~d} \theta+\frac{4}{\gamma} \int_{-\pi}^{\pi} \int_{I}\left|\xi^{\prime}(r)\right|^{2}|\phi(r, \theta)|^{2} r \mathrm{~d} r \mathrm{~d} \theta+2 \int_{-\pi}^{\pi} \int_{I}|\phi(r, \theta)|^{2} r \mathrm{~d} r \mathrm{~d} \theta \\
& \leq \frac{4}{\gamma} \int_{-\pi}^{\pi} \int_{0}^{\infty}\left|\partial_{r} \phi(r, \theta)\right|^{2} r \mathrm{~d} r \mathrm{~d} \theta+\left(\frac{4}{\gamma}\left\|\xi^{\prime}\right\|_{\infty}^{2}+2\right) \int_{-\pi}^{\pi} \int_{I}|\phi(r, \theta)|^{2} r \mathrm{~d} r \mathrm{~d} \theta
\end{aligned}
$$

Coming back to the test function $\psi$ and recalling (34), we have therefore proved

$$
\int_{\mathbb{R}^{2}}\left|\nabla_{A} \psi(x)\right|^{2} \mathrm{~d} x \geq \frac{\gamma}{4} \int_{\mathbb{R}^{2}} \frac{|\psi(x)|^{2}}{1+|x|^{2} \log ^{2}\left(|x| / r_{0}\right)} \mathrm{d} x-\left(\|\xi\|_{\infty}^{2}+\frac{\gamma}{2}\right) \int_{\mathbb{R}^{2}} \chi_{I}(x)|\psi(x)|^{2} \mathrm{~d} x .
$$

Finally, interpolating between (33) and (37), we get

$$
\int_{\mathbb{R}^{2}}\left|\nabla_{A} \psi(x)\right|^{2} \mathrm{~d} x \geq\left[(1-\varepsilon) \nu-\varepsilon\left(\|\xi\|_{\infty}^{2}+\frac{\gamma}{2}\right)\right] \int_{\mathbb{R}^{2}} \chi_{I}(x)|\psi(x)|^{2} \mathrm{~d} x+\varepsilon \frac{\gamma}{4} \int_{\mathbb{R}^{2}} \frac{|\psi(x)|^{2}}{1+|x|^{2} \log ^{2}\left(|x| / r_{0}\right)} \mathrm{d} x
$$

with any $\varepsilon>0$. Choosing $\varepsilon$ in such a way that the square bracket vanishes, we obtain (32) with

$$
\tilde{c} \geq \frac{\frac{\gamma}{4} \nu}{\nu+\|\xi\|_{\infty}^{2}+\frac{\gamma}{2}} \inf _{r \in(0, \infty)} \frac{1+r^{2} \log ^{2}(r)}{1+r^{2} \log ^{2}\left(r / r_{0}\right)}>0
$$

The theorem is proved.

Now we are in a position to prove Theorem 1, It follows as a consequence of (26) and Theorem 4 under the stronger hypothesis (22).

Proof of Theorem 1. The claim follows by mimicking the proof of [4, Thm. 3.2].

In view of the hypothesis (1) and since $B$ is supposed to have a compact support, there exists a positive number $R$ such that (31) holds for all $r \geq R$. Indeed, it is enough to choose $R$ so large that the support of $B$ is contained in the disk $D_{R}$. As a consequence of Theorem 4 , we therefore obtain

$$
\int_{\mathbb{R}^{2}}\left|\nabla_{A} \psi(x)\right|^{2} \mathrm{~d} x \geq \tilde{c} \int_{D_{R}} \frac{|\psi(x)|^{2}}{1+|x|^{2} \log ^{2}(|x|)} \mathrm{d} x \geq \tilde{c} a_{R} \int_{D_{R}} \frac{|\psi(x)|^{2}}{1+|x|^{2}} \mathrm{~d} x,
$$

where $\tilde{c}$ is positive and

$$
a_{R}:=\inf _{r \in(0, R)} \frac{1+r^{2}}{1+r^{2} \log ^{2}(r)}
$$

is also a positive constant.

At the same time, recalling that the function $r \mapsto \lambda_{a}(r)$ is constant on $[R, \infty)$, the local Hardy inequality (26) yields

$$
\int_{\mathbb{R}^{2}}\left|\nabla_{A} \psi(x)\right|^{2} \mathrm{~d} x \geq \lambda_{a}(R) \int_{\mathbb{R}^{d} \backslash D_{R}} \frac{|\psi(x)|^{2}}{|x|^{2}} \mathrm{~d} x \geq \lambda_{a}(R) \int_{\mathbb{R}^{d} \backslash D_{R}} \frac{|\psi(x)|^{2}}{1+|x|^{2}} \mathrm{~d} x,
$$

where $\lambda_{a}(R)$ is positive due to Lemma 1.

Combining the two inequalities (38) and (39), we get (21) with $c \geq \min \left\{\tilde{c} a_{R}, \lambda_{a}(R)\right\}>0$. 
Remark 5. The Hardy inequality of Theorem 1 is optimal in the sense that if the hypothesis (1) does not hold, then (2) cannot hold with a positive constant $c$, namely

$$
c_{*}:=\inf _{\substack{\psi \in C_{0}^{\infty}\left(\mathbb{R}^{2}\right) \\ \psi \neq 0}} \frac{\int_{\mathbb{R}^{2}}\left|\nabla_{A} \psi(x)\right|^{2} \mathrm{~d} x}{\int_{\mathbb{R}^{2}} \frac{|\psi(x)|^{2}}{1+|x|^{2}} \mathrm{~d} x}=0 .
$$

To see it, let us pass to the polar coordinates as above. We set $\phi(r, \theta):=f(r) \varphi_{R}(\theta)$, where $R$ is such that $D_{R} \supset \operatorname{supp} B, f$ is such that $\operatorname{supp} f \subset(R, \infty)$ and $\varphi_{R}$ is the eigenfunction of the operator $P_{a(R, \cdot)^{*}} P_{a(R, \cdot)}$ corresponding to the eigenvalue $\lambda_{a}(R), c f$. (25). If (1) is violated, then $\lambda_{a}(R)=0$ due to Lemma 1 ] Consequently,

$$
c_{*} \leq \inf _{\substack{f \in C_{0}^{\infty}((R, \infty)) \\ f \neq 0}} \frac{\int_{0}^{\infty}\left|f^{\prime}(r)\right|^{2} r \mathrm{~d} r}{\int_{0}^{\infty} \frac{|f(r)|^{2}}{1+|r|^{2}} r \mathrm{~d} r} .
$$

It is well known that this infimum equals zero. An explicit minimising sequence is for example given by a mollification of

$$
f_{n}(r):=\frac{1}{\log n} \begin{cases}\log (r / n) & \text { if } r \in\left[n, n^{2}\right] \\ \log \left(n^{3} / r\right) & \text { if } r \in\left[n^{2}, n^{3}\right] \\ 0 & \text { otherwise }\end{cases}
$$

with $n>R$.

\subsection{The Aharonov-Bohm field}

Finally, we establish Theorem 2 dealing with the vector potential (5).

Proof of Theorem [. Let $\psi \in C_{0}^{\infty}\left(\mathbb{R}^{2} \backslash\{0\}\right)$. Passing to the polar coordinates as in (24) and proceeding as in (26), we have

$$
\begin{aligned}
\int_{\mathbb{R}^{2}}\left|\nabla_{A_{\infty}} \psi(x)\right|^{2} \mathrm{~d} x & =\int_{0}^{\infty} \int_{-\pi}^{\pi}\left(\left|\partial_{r} \phi(r, \theta)\right|^{2}+\frac{\left|-i \partial_{\theta} \phi(r, \theta)-\alpha \phi(r, \theta)\right|^{2}}{r^{2}}\right) \mathrm{d} \theta r \mathrm{~d} r \\
& \geq \int_{0}^{\infty} \int_{-\pi}^{\pi} \frac{\lambda_{\alpha}}{r^{2}}|\phi(r, \theta)|^{2} \mathrm{~d} \theta r \mathrm{~d} r \\
& =\lambda_{\alpha} \int_{\mathbb{R}^{2}} \frac{|\psi(x)|^{2}}{|x|^{2}} \mathrm{~d} x .
\end{aligned}
$$

The only (but significant) difference with respect to smooth non-trivial fields is that $\alpha$ is a constant (so in particular independent of the radial coordinate $r$ ), and therefore $\lambda_{\alpha}$ is constant. By virtue of Lemma 1. $\lambda_{\alpha}$ is positive if, and only if, the flux condition (6) holds. This concludes the proof of Theorem 2 with $c_{\infty} \geq \lambda_{\alpha}$

\subsection{Robust Hardy inequalities}

In this subsection we go beyond the special choice (3) of the vector potential. Our aim is to establish a Hardy inequality valid for any smooth choice of the vector potential $A$ whenever the complex-valued magnetic field $B$ is non-trivial (and the imaginary part satisfies some extra condition). We are inspired by the gauge-free proof of [4, Thm. 1.1], but important modifications are needed because the diamagnetic inequality is not available for complex-valued magnetic fields.

Given a smooth complex field $B: \mathbb{R}^{2} \rightarrow \mathbb{C}$, let $A: \mathbb{R}^{2} \rightarrow \mathbb{C}^{2}$ be any smooth vector potential satisfying $\operatorname{rot} A=B$. For every positive number $R$, we introduce the non-negative quantity

$$
\mu_{A}(R):=\inf _{\substack{\psi \in C^{\infty}\left(\overline{D_{R}}\right) \\ \psi \neq 0}} \frac{\int_{D_{R}}\left|\nabla_{A} \psi(x)\right|^{2} \mathrm{~d} x}{\int_{D_{R}}|\psi(x)|^{2} \mathrm{~d} x} .
$$


The following lemma ensures that the function $R \mapsto \mu_{A}(R)$ is identically equal to zero if, and only if, the magnetic field $B$ is trivial in $\mathbb{R}^{2}$.

Lemma 2. Given any $R \in(0, \infty), \mu_{A}(R)=0$ if, and only if, $B=0$ on $D_{R}$.

Proof. The number $\mu_{A}(R)$ as defined in (40) is the spectral threshold of the (self-adjoint) magnetic Schrödinger operator $\left(\nabla_{A}\right)^{*} \nabla_{A}$ in $L^{2}\left(D_{R}\right)$, subject to magnetic Neumann boundary conditions. Since $A$ is bounded on the disk $D_{R}$, the form domain coincides with the Sobolev space $W^{1,2}\left(D_{R}\right)$, which is compactly embedded in $L^{2}\left(D_{R}\right)$. Hence the spectrum of the operator is purely discrete, the infimum in (40) is achieved and $\mu_{A}(R)$ is the lowest eigenvalue of the operator. Let us denote by $\psi_{R}$ a corresponding eigenfunction, which is smooth due to elliptic regularity theory.

If $\mu_{A}(R)=0$, then $\nabla_{A} \psi_{R}=0$ in $D_{R}$, which is equivalent to the system of two equations

$$
\begin{aligned}
& -i \partial_{1} \psi_{R}-A_{1} \psi_{R}=0, \\
& -i \partial_{2} \psi_{R}-A_{2} \psi_{R}=0,
\end{aligned}
$$

where we write $A=\left(A_{1}, A_{2}\right)$. Differentiating the first equation with respect to the second variable, differentiating the second equation with respect to the first variable and subtracting the two results, we arrive at the pointwise identity

$$
B \psi_{R}=0 \quad \text { on } \quad D_{R} .
$$

At the same time, differentiating the first equation of (41) with respect to the first variable, differentiating the second equation with respect to the second variable and adding the two results, we obtain that $\psi_{R}$ satisfies the stationary Schrödinger equation

$$
-\Delta \psi_{R}+\left(i \operatorname{div} A-A^{2}\right) \psi_{R}=0 \quad \text { in } \quad D_{R},
$$

where $A^{2}:=A_{1}^{2}+A_{2}^{2}$. Taking into account that (41) also implies that if $\psi_{R}$ vanishes at a point of $D_{R}$ then it vanishes there of infinite order, the unique continuation property ensures that $\psi_{R}$ is nowhere zero in $D_{R}$. Hence, (42) implies that $B=0$ in $D_{R}$.

To prove the opposite implications, let us assume that $B=0$ in $D_{R}$ (i.e. $A$ is closed). Then there exists a smooth function $F: \mathbb{R}^{2} \rightarrow \mathbb{C}$ such that $A=\nabla F$ (i.e. $A$ is exact). Choosing $\psi=e^{i F}$ as the test function in the variational definition (40), we obtain $\mu_{A}(R)=0$.

The following theorem is a variant of Theorem 4 obtained under alternative hypotheses. It is also a complex extension of the robust magnetic Hardy inequality [4, Thm. 1.1].

Theorem 5. Let a smooth complex field $B: \mathbb{R}^{2} \rightarrow \mathbb{C}$ be not identically equal to zero. Then, for any smooth vector potential $A: \mathbb{R}^{2} \rightarrow \mathbb{C}^{2}$ satisfying $B=\operatorname{rot} A$ and the hypothesis

$$
\lim _{|x| \rightarrow \infty}(|\Im A(x)||x| \log (|x|))=0,
$$

there exists a positive constant $\hat{c}$ depending on A such that the following inequality holds

$$
\forall \psi \in C_{0}^{\infty}\left(\mathbb{R}^{2}\right), \quad \int_{\mathbb{R}^{2}}\left|\nabla_{A} \psi(x)\right|^{2} \mathrm{~d} x \geq \hat{c} \int_{\mathbb{R}^{2}} \frac{|\psi(x)|^{2}}{1+|x|^{2} \log ^{2}(|x|)} \mathrm{d} x .
$$

Proof. We are inspired by the gauge-free proof of [4, Thm. 1.1].

Let $\psi \in C_{0}^{\infty}\left(\mathbb{R}^{2}\right)$. The definition (40) yields

$$
\int_{\mathbb{R}^{2}}\left|\nabla_{A} \psi(x)\right|^{2} \mathrm{~d} x \geq \mu_{A}(R) \int_{D_{R}}|\psi(x)|^{2} \mathrm{~d} x,
$$

where $\mu_{A}(R)$ is positive for all sufficiently large $R$ whenever $B$ is non-trivial. This is a variant of the local Hardy inequality (33) with $I:=(0, R)$. To conclude with the global Hardy inequality (44) by mimicking the rest of the proof of Theorem 4 however, we would also need (34). For purely real-valued vector potentials considered in [4, a variant of (34) (with the absolute value $|\phi|$ instead of $\phi$ ) follows by the diamagnetic inequality [23, Thm. 7.21]. In our more general case, this inequality only yields

$$
\forall x \in \mathbb{R}^{2}, \quad\left|\nabla_{A} \psi(x)\right| \geq\left|\nabla_{\Im A}\right| \psi(x)||,
$$


and we have to proceed differently.

Set $r_{0}:=R / 2$, the middle point of the interval $I$. Suppose for a moment that $\psi \in C_{0}^{\infty}\left(\mathbb{R}^{2} \backslash \overline{D_{r_{0}}}\right)$. Then, for every real constant $\beta$, we have

$$
\begin{aligned}
0 \leq & \int_{\mathbb{R}^{2}}\left|\nabla_{\Im A}\right| \psi(x)\left|-\beta \frac{x}{|x|^{2} \log \left(|x| / r_{0}\right)}\right| \psi(x)||^{2} \mathrm{~d} x \\
= & \left.\int_{\mathbb{R}^{2}}\left|\nabla_{\Im A}\right| \psi(x)\right|^{2} \mathrm{~d} x+\beta^{2} \int_{\mathbb{R}^{2}} \frac{|\psi(x)|^{2}}{|x|^{2} \log ^{2}\left(|x| / r_{0}\right)} \mathrm{d} x \\
& \quad-\beta \int_{\mathbb{R}^{2}} \frac{x \cdot \nabla|\psi(x)|^{2}}{|x|^{2} \log \left(|x| / r_{0}\right)} \mathrm{d} x-2 \beta \int_{\mathbb{R}^{2}} \frac{x \cdot \Im A(x)}{|x|^{2} \log \left(|x| / r_{0}\right)}|\psi(x)|^{2} \mathrm{~d} x \\
= & \left.\int_{\mathbb{R}^{2}}\left|\nabla_{\Im A}\right| \psi(x)\right|^{2} \mathrm{~d} x+\left(\beta^{2}-\beta\right) \int_{\mathbb{R}^{2}} \frac{|\psi(x)|^{2}}{|x|^{2} \log ^{2}\left(|x| / r_{0}\right)} \mathrm{d} x-2 \beta \int_{\mathbb{R}^{2}} \frac{x \cdot \Im A(x)}{|x|^{2} \log \left(|x| / r_{0}\right)}|\psi(x)|^{2} \mathrm{~d} x .
\end{aligned}
$$

Here the second equality follows by an integration by parts. Recalling (46) and denoting

$$
k_{R}:=\sup _{|x| \geq r_{0}}\left(|\Im A(x)||x| \log \left(|x| / r_{0}\right)\right),
$$

which tends to zero as $R \rightarrow \infty$ due to (43), we have thus obtained the lower bound

$$
\begin{aligned}
\int_{\mathbb{R}^{2}}\left|\nabla_{A} \psi(x)\right|^{2} \mathrm{~d} x & \geq\left(-\beta^{2}+\beta-2|\beta| k_{R}\right) \int_{\mathbb{R}^{2}} \frac{|\psi(x)|^{2}}{|x|^{2} \log ^{2}\left(|x| / r_{0}\right)} \mathrm{d} x \\
& =\gamma_{R} \int_{\mathbb{R}^{2}} \frac{|\psi(x)|^{2}}{|x|^{2} \log ^{2}\left(|x| / r_{0}\right)} \mathrm{d} x \quad \text { with } \quad \gamma_{R}:=\left(\frac{1-2 k_{R}}{2}\right)^{2} .
\end{aligned}
$$

Here the equality follows by the best choice $\beta:=\left(1-2 k_{R}\right) / 2$, assuming that $R$ is so large that $k_{R}<1 / 2$.

To employ the estimate (47) for our test function $\psi \in C_{0}^{\infty}\left(\mathbb{R}^{2}\right)$, we use the cut-off $\xi$ from the proof of Theorem 4. Similarly as in (36), we have

$$
\begin{aligned}
\int_{\mathbb{R}^{2}} & \frac{|\psi(x)|^{2}}{1+|x|^{2} \log ^{2}\left(|x| / r_{0}\right)} \mathrm{d} x \\
& \leq 2 \int_{\mathbb{R}^{2} \backslash D_{r_{0}}} \frac{|\xi(|x|) \psi(x)|^{2}}{|x|^{2} \log ^{2}\left(|x| / r_{0}\right)} \mathrm{d} x+2 \int_{D_{r_{0}}}|\xi(|x|) \psi(x)|^{2} \mathrm{~d} x+2 \int_{\mathbb{R}^{2}}|(1-\xi(|x|)) \psi(x)|^{2} \mathrm{~d} x \\
& \leq \frac{2}{\gamma_{R}} \int_{\mathbb{R}^{2} \backslash D_{r_{0}}}\left|\nabla_{A}(\xi(|x|) \psi(x))\right|^{2} \mathrm{~d} x+2 \int_{D_{r_{0}}}|\psi(x)|^{2} \mathrm{~d} x+2 \int_{D_{R}}|\psi(x)|^{2} \mathrm{~d} x \\
& \leq \frac{4}{\gamma_{R}} \int_{\mathbb{R}^{2} \backslash D_{r_{0}}}|\xi(|x|)|^{2}\left|\nabla_{A} \psi(x)\right|^{2} \mathrm{~d} x+\frac{4}{\gamma_{R}} \int_{\mathbb{R}^{2} \backslash D_{r_{0}}}\left|\xi^{\prime}(|x|)\right|^{2}|\psi(x)|^{2} \mathrm{~d} x+4 \int_{D_{R}}|\psi(x)|^{2} \mathrm{~d} x \\
& \leq \frac{4}{\gamma_{R}} \int_{\mathbb{R}^{2} \backslash D_{r_{0}}}\left|\nabla_{A} \psi(x)\right|^{2} \mathrm{~d} x+\left(\frac{4}{\gamma_{R}}\left\|\xi^{\prime}\right\|_{\infty}^{2}+4\right) \int_{D_{R}}|\psi(x)|^{2} \mathrm{~d} x,
\end{aligned}
$$

where we have used (47) in the second inequality. In analogy with (37), we have therefore proved

$$
\int_{\mathbb{R}^{2}}\left|\nabla_{A} \psi(x)\right|^{2} \mathrm{~d} x \geq \frac{\gamma_{R}}{4} \int_{\mathbb{R}^{2}} \frac{|\psi(x)|^{2}}{1+|x|^{2} \log ^{2}\left(|x| / r_{0}\right)} \mathrm{d} x-\left(\|\xi\|_{\infty}^{2}+\gamma_{R}\right) \int_{D_{R}}|\psi(x)|^{2} \mathrm{~d} x .
$$

As in the end of the proof of Theorem 4 interpolating between (45) and (48), we get

$$
\int_{\mathbb{R}^{2}}\left|\nabla_{A} \psi(x)\right|^{2} \mathrm{~d} x \geq\left[(1-\varepsilon) \mu_{A}(R)-\varepsilon\left(\|\xi\|_{\infty}^{2}+\gamma_{R}\right)\right] \int_{D_{R}}|\psi(x)|^{2} \mathrm{~d} x+\varepsilon \frac{\gamma_{R}}{4} \int_{\mathbb{R}^{d}} \frac{|\psi(x)|^{2}}{1+|x|^{2} \log ^{2}\left(|x| / r_{0}\right)} \mathrm{d} x
$$

with any $\varepsilon>0$. Choosing $\varepsilon$ in such a way that the square bracket vanishes, we obtain (44) with

$$
\hat{c} \geq \frac{\frac{\gamma_{R}}{4} \mu_{A}(R)}{\mu_{A}(R)+\|\xi\|_{\infty}^{2}+\gamma_{R}} \inf _{r \in(0, \infty)} \frac{1+r^{2} \log ^{2}(r)}{1+r^{2} \log ^{2}\left(r / r_{0}\right)}>0 .
$$

The theorem is proved. 
Remark 6. The hypothesis (43) is clearly a condition on the rate of decay of $\Im A$ at infinity. Such kind of condition would not be acceptable for the real part of $A$ (because of the gauge invariance), but it seems to be a relevant assumption when the imaginary part of $A$ is present. On the other hand, it is just a sufficient condition to make the proof of Theorem 5 work. An alternative to (47) is to integrate by parts and get the identity

$$
\begin{aligned}
\int_{\mathbb{R}^{2}}\left|\nabla_{\Im A} \psi(x)\right|^{2} \mathrm{~d} x & =\int_{\mathbb{R}^{2}}|\nabla| \psi(x)||^{2} \mathrm{~d} x+\int_{\mathbb{R}^{2}}|\Im A(x)|^{2}|\psi(x)|^{2} \mathrm{~d} x+\int_{\mathbb{R}^{2}} \Im A(x) \cdot \nabla|\psi(x)|^{2} \mathrm{~d} x \\
& =\int_{\mathbb{R}^{2}}|\nabla| \psi(x)||^{2} \mathrm{~d} x+\int_{\mathbb{R}^{2}}\left(|\Im A(x)|^{2}-\operatorname{div} \Im A(x)\right)|\psi(x)|^{2} \mathrm{~d} x
\end{aligned}
$$

valid for all $\psi \in C_{0}^{\infty}\left(\mathbb{R}^{2}\right)$. If $|\Im A|^{2}-\operatorname{div} \Im A$ is non-negative, then one immediately gets (34) with help of (46) and one can proceed as in the proof of Theorem 4 (with (45) instead of (33)). In fact, it is enought to assume that $|\Im A|^{2}-\operatorname{div} \Im A$ is non-negative outside the disk $D_{R}$ where $R$ can be chosen arbitrarily large. Yet another possibility is to replace the sign condition by the asymptotic behaviour

$$
\lim _{|x| \rightarrow \infty}\left[\left(|\Im A|^{2}-\operatorname{div} \Im A\right)_{-}(x)|x|^{2} \log ^{2}(|x|)\right]=0,
$$

where $f_{-}$denotes the negative part of a function $f$.

\section{Acknowledgment}

The author was partially supported by the GACR grant No. 18-08835S and by FCT (Portugal) through project PTDC/MAT-CAL/4334/2014.

\section{References}

[1] N. Ananikian and R. Kenna, Imaginary magnetic fields in the real world, Physics 8 (2015), 2.

[2] M. Astudillo, P. Kurasov, and M. Usman, RJ-symmetric Laplace operators on star graphs: Real spectrum and self-adjointness, Adv. Math. Phys. (2015), 649795.

[3] C. M. Bender and P. N. Boettcher, Real spectra in non-Hermitian Hamiltonians having PT symmetry, Phys. Rev. Lett. 80 (1998), 5243-5246.

[4] C. Cazacu and D. Krejčiř́k, The Hardy inequality and the heat equation with magnetic field in any dimension, Comm. Partial Differential Equations 41 (2016), 1056-1088.

[5] E. B. Davies, Linear operators and their spectra, Cambridge University Press, 2007.

[6] J. Dieudonné, Quasi-Hermitian operators, Proceedings of the International Symposium on Linear Spaces (Jerusalem 1960), Jerusalem Academic Press, Pergamon, Oxford, 1961, pp. 115-123.

[7] E. Ergun, A two-parameter family of non-Hermitian Hamiltonians with real spectrum, J. Phys. A: Math. Theor. 43 (2010), 455212.

[8] _ Finding of the metric operator for a quasi-Hermitian model, J. Funct. Spaces Appl. 2013 (2013), 716109 .

[9] E. Ergun and M. Saglam, On the metric of a non-Hermitian model, Rep. Math. Phys. 65 (2010), 367378 .

[10] I. C. Gohberg and M. G. Krein, Introduction to the theory of linear nonselfadjoint operators in Hilbert space, Amer. Math. Soc., 1969.

[11] N. Hatano and D. R. Nelson, Localization transitions in non-Hermitian quantum mechanics, Phys. Rev. Lett. 77 (1996), 570-573.

[12] _ Non-Hermitian delocalization and eigenfunctions, Phys. Rev. B 58 (1998), 8384-8390. 
[13] A. Hussein, D. Krejčiř́ík, and P. Siegl, Non-self-adjoint graphs, Trans. Amer. Math. Soc. 367 (2015), 2921-2957.

[14] T. Kato, Perturbation theory for linear operators, Springer-Verlag, Berlin, 1966.

[15] D. Krejčiřík, Calculation of the metric in the hilbert space of a $\mathcal{P T}$-symmetric model via the spectral theorem, J. Phys. A: Math. Theor. 41 (2008), 244012.

[16] D. Krejčiřík, The improved decay rate for the heat semigroup with local magnetic field in the plane, Calc. Var. Partial Differ. Equ. 47 (2013), 207-226.

[17] D. Krejčiř́ik, H. Bíla, and M. Znojil, Closed formula for the metric in the Hilbert space of a $\mathcal{P} \mathcal{T}$ symmetric model, J. Phys. A 39 (2006), 10143-10153.

[18] D. Krejčiř́ik and P. Siegl, Elements of spectral theory without the spectral theorem, In Non-selfadjoint operators in quantum physics: Mathematical aspects (432 pages), F. Bagarello, J.-P. Gazeau, F. H. Szafraniec, and M. Znojil, Eds., Wiley-Interscience, 2015.

[19] D. Krejčiř́́k, P. Siegl, M. Tater, and J. Viola, Pseudospectra in non-Hermitian quantum mechanics, J. Math. Phys. 56 (2015), 103513.

[20] D. Krejčiřík, P. Siegl, and Železný, On the similarity of Sturm-Liouville operators with nonHermitian boundary conditions to self-adjoint and normal operators, Complex Anal. Oper. Theory 8 (2014), 255-281.

[21] P. Kurasov and B. M. Garjani, Quantum graphs: PT-symmetry and reflection symmetry of the spectrum, J. Math. Phys. 58 (2017), 023506.

[22] A. Laptev and T. Weidl, Hardy inequalities for magnetic Dirichlet forms, Oper. Theory Adv. Appl. 108 (1999), 299-305.

[23] E. H. Lieb and M. Loss, Analysis, American Mathematical Society, Providence, Rhode Island, 1997.

[24] X. Peng, H. Zhou, B.-B. Wei, J. Cui, J. Du, and R.-B. Liu, Experimental observation of Lee-Yang zeros, Phys. Rev. Lett. 114 (2015), 010601.

[25] N. Raymond, Bound states of the magnetic Schrödinger operator, EMS, Zürich, 2017.

[26] F. G. Scholtz, H. B. Geyer, and F. J. W. Hahne, Quasi-Hermitian operators in quantum mechanics and the variational principle, Ann. Phys 213 (1992), 74-101.

[27] P. Siegl, Supersymmetric quasi-Hermitian Hamiltonians with point interactions on a loop, J. Phys. A: Math. Theor. 41 (2008), 244025. 